\title{
ZU NEUESTEN ENTWICKLUNGSTENDENZEN VON BILDUNGSMEDIEN: MODERNE UND ALTE SCHULBÜCHER - EIN KURZER WEG VOM LEHRBUCH ZUM BILDUNGSMEDIUM
}

\begin{abstract}
Das Ziel des Beitrags ist es, die Entwicklung des Mediums Schulbuch / Lehrbuch zum modernen Bildungsmedium / Lehrwerk in den letzten dreißig-vierzig Jahren am Beispiel von Lehrmitteln zum Erlernen des Deutschen als Fremdsprache zu skizzieren. In den meisten Fällen stellt heute ein Fremdsprachenlehrwerk eine multimodale Medienkombination dar. Es kann auch als ein intermediales Phänomen betrachtet werden. Ausgegangen von einem vor 30 Jahren in den meisten polnischen Schulen eingesetzten Lehrbuch Lernt mit uns, welches für ein ,didaktisches Massenmedium' der 80er Jahre in Polen gehalten werden kann, wird die Entwicklung der Fremdsprachenlehrwerke für den DaF-Unterricht präsentiert. $\mathrm{Zu}$ diesem Zweck wird das genannte Lehrbuch aus den 80er Jahren mit einem modernen Lehrwerk für $\mathrm{DaF}$ Infos kontrastiert. Die größten (und bedeutendsten) Veränderungen beziehen sich auf die Entwicklung des bikodalen Buches zu einem mehrteiligen multikodalen Gefüge. Von Interesse sind nicht nur die Veränderungen, die die Lehrmittel in den letzten drei Jahrzehnten in Bezug auf ihr Aussehen, ihren Inhalt und Aufbau erfahren haben. Gefragt wird auch nach dem aktuellen Status und der Eigenart des modernen Lehrwerks, wobei seine Multimodalität, , Medienhaftigkeit" und intermediale Bezüge in den Vordergrund gestellt werden. Im Beitrag wird auch auf die bisherige linguistische (germanistische) Schulbuchforschung eingegangen. Es werden ebenfalls einige Forschungsdesiderate formuliert.
\end{abstract}

Schlüsselwörter: Schulbuch, Lehrwerk, Multimedium, Multimodalität, intermediale Bezüge, Fremdsprachenlernen, DaF, DaF-Unterricht.

\section{EINLEITUNG}

Im vorliegenden Beitrag möchte ich einige Besonderheiten in der Entwicklung von Schulbüchern und insbesondere von Fremdsprachenlehrwerken skizzieren, um anhand historischer Entwicklung ihren aktuellen Status zu hinterfragen und die modernen Entwicklungstendenzen aufzuzeigen. In meinen Ausführungen stütze ich mich einerseits auf einschlägige Literatur und exemplarische Analyse von einigen Lehrmitteln, andererseits beziehe ich mich auf meine berufliche Erfahrung als Fremdsprachendidaktikerin sowie auf meine persönliche Erfahrung als Lernende. Schulbücher, darunter auch Fremdsprachenlehrwerke, sind Massenmedien besonderer Art. Jeder hat sie, zumindest in der eigenen Schulzeit, in Anspruch genommen und aus ihnen gelernt. Mein erstes Deutschlehrbuch Lernt mit

\footnotetext{
${ }^{1}$ Dr Marta Smykała, adiunkt, Katedra Lingwistyki Stosowanej, Wydział Filologiczny, Uniwersytet Rzeszowski; e-mail: smykala@intertele.pl
} 
uns von J. Honsza und N. Honsza ${ }^{2}$ war in den 80er Jahren nicht nur ein, sondern das didaktische Massenmedium, das in den Mittelschulen in ganz Polen im Einsatz war. Das Buch hatte eine bunte Umschlagseite, im Inneren war es größtenteils schwarz-weiß. Nur noch eine Farbe gab es in jedem der vier Bände zusätzlich. Mithilfe dieser einen anderen Farbe waren Aufgabenbeschreibungen und wichtigere Regeln abgebildet. Ähnliches Gestaltungsprinzip galt auch für andere Lehrbücher, darunter auch für Bücher aus dem Ausland (DDR): farbiger Einband, das Innere schwarz-weiß, kaum Bilder. Führt man sich - im Kontrast dazu - die neuen Lehr- und Lernmaterialien vor Augen, ist der Anteil des Visuellen und der Einsatz von Farben der erste augenfälligste Unterschied. Der zweite Unterschied ist quantitativer Natur und bezieht sich auf die Anzahl der Artefakte, die man beim Lernen nutzt. In den 80er Jahren war Lernt mit uns nicht nur ein Leitmedium im Unterricht, sondern es war überhaupt das einzige Medium, in dem Schritt für Schritt, eine Übung nach der anderen durchgenommen wurde. Die alten Lehrbücher bestanden eben meistens aus dem einem Buch und je nach Bedarf griff man noch nach separaten Grammatiken, anderen Büchern, die aber in keinerlei Beziehung zu dem jeweiligen Lehrbuch standen, abgesehen davon, dass sie auch für Deutschlernende konzipiert waren. Heute ist ein modernes Fremdsprachenlehrwerk eine multimodale Medienkombination (Medienverbund). Es kann auch als ein intermediales Phänomen betrachtet werden im Sinne eines Multimediums, das meistens aus einigen Büchern und CD(s), sowie DVD(s) und einem Online-Angebot für Schüler und Lehrer besteht. Immer häufiger kommt noch ein Zusatzangebot hinzu: Übungen und Aktivitäten für den Unterricht, die mittels eines verhältnismäßig neuen Mediums im Schulbereich, nämlich des interaktiven Whiteboards (IWB) ausgeführt werden. Außerdem gibt es viele außerschulische Formen und Foren, Techniken und Methoden, die z. B. als Freizeitangebot online zugänglich sind (z. B. verschiedene Webinars, YouTube-Angebot, oder SITA-Brille mit Atemfeedback-Technik, um nur einige wenige zu nennen).

Ist also der Einsatz von Visuellem sowie von Medien-Paketen und vernetzten Textsorten zum Erlernen einer Fremdsprache eine Erfindung der letzten vierzig Jahre? Auf keinen Fall. Zum Bildeinsatz bemerkt Adamzik mit Recht, dass man sich spätestens seit 1635, d.h. seit Comenius und seinem Werk Orbis sensualium pictus, der Bedeutung von Bildern im Unterrichtsprozess bewusst ist ${ }^{3}$. Der Farbdruck ist, zugegeben, eine spätere Erfindung. In Bezug auf das, was heute in Form von einem medialen Gefüge zustande kommt, kann festgestellt werden, dass es seine Vorläufer schon im 17. und 18. Jahrhundert hatte. Damals ,hatte eine ,Grammatik' als Sprachlehrbuch offensichtlich auch noch andere Aufgaben zu erfüllen. Zumeist als Anhang angeschlossene Alltagsgespräche, Sprichwörtersammlungen, Musterbriefe und nicht zuletzt Anekdoten sowie längere Erzählungen dienten der Schulung sprachlicher Fertigkeiten, sie lieferten den Stoff für Übersetzungsübungen und wurden auch bei der Ausspracheschulung und im Literaturunterricht eingesetzt ${ }^{* 4}$. Als ein Zwischenfazit kann daher gelten, dass bereits vor einigen Jahrhunderten das Bewusstsein vorhanden war, dass der Fremdsprachenerwerb die Entwicklung von verschiedenen sprachlichen Fertigkeiten umfasst, sich auf vielfältige Textsorten und authentische Texte stützen soll, sowie auf kulturkundliche Inhalte und Bilder zwecks besserer Visualisierung nicht verzichten kann

2 J. Honsza, N. Honsza, Lernit mit uns 1, 2, 3, 4, Warszawa 1979.

${ }^{3}$ Vgl. K. Adamzik, Kontrastive Textologie am Beispiel des Schulbuchs. In: tekst i dyskurs / text und diskurs 5, 2012, S. 55.

${ }^{4}$ R. Budziak, Deutsch als Fremdsprache in Polen. Sprachlehrbücher aus dem 16. bis 18. Jahrhundert, Harrasowitz Verlag, Wiesbaden 2011, S. 55. 
(mehr zu alten Sprachlehrbüchern in Polen vgl. exemplarisch Zawadzka $2005^{5}$ und Budziak $\left.2011^{6}\right)$. Die genannten Prinzipien gelten auch in der modernen Didaktik.

\section{SCHULBUCH IN DER LINGUISTISCHEN FORSCHUNG}

Bevor ich meine Ausführungen fortsetze, will ich darauf hinweisen, dass hier die Bezeichnungen Fremdsprachlehrwerk (Fremdsprachenlehrbuch), Sprachlehrbuch und Schulbuch abwechselnd im Sinne von Lehr- und Lernmitteln verwendet werden. All die Bezeichnungen sind eng verwandt, wenn auch nicht ganz synonymisch zu verstehen. Schulbuch ist ein Oberbegriff, der sowohl die Bezeichnung Fremdsprachenlehrbuch als auch Fremdsprachenlehrwerk umfasst. Die Bezeichnung Schulbuch bezieht sich auf den institutionellen Rahmen. Lehrbuch betont die Tätigkeit des Lehrens. In den Bezeichnungen Lehr-Buch und Lehr-Werk spiegelt sich bereits die Entwicklung des Untersuchungsgegenstands wider. Die Bezeichnung Lehrwerk visiert an, dass es aus mehreren Teilen (Büchern u. anderen Komponenten) besteht. Was in Bezug auf das Schulbuch die linguistische Forschung bis jetzt erforscht hat, gilt fast uneingeschränkt auch für Fremdsprachenlehrbücher / Fremdsprachenlehrwerke. Gewisse Einschränkungen ergeben sich aus der Tatsache, dass Schulbücher bestimmte Genehmigungsverfahren erfahren müssen, bevor sie in der Schule eingesetzt werden. In der Tat sind nicht alle Fremdsprachenlehrwerke zum schulischen Einsatz zugelassen. Für meine weiteren Ausführungen hat dies aber keine Relevanz.

Welche Aufschlüsse über die linguistische (germanistische) ${ }^{7}$ Schulbuchforschung gibt die Lektüre von einschlägiger Literatur? In der Fachliteratur dominiert die Auffassung, dass innerhalb der germanistischen Linguistik das Schulbuch als Untersuchungsgegenstand derzeit erst ,entdeckt wird“8 ${ }^{\circ}$ und ,die germanistisch-linguistische Forschung hierzu insgesamt defizitär ausfällt"“9 . Die aktuellen Forschungsdomänen gestalten sich je nach der Perspektive, aus welcher das Schulbuch betrachtet wird.

Grundsätzlich lassen sich gegenwärtig drei Perspektiven und damit drei linguistische Forschungsfelder unterscheiden: Erstens kann das Schulbuch als Text bzw. Textsorte oder Textsortenverbund und damit ein Untersuchungsgegenstand der Textlinguistik wahrgenommen werden. In diesem Kontext sei u.a. auf Arbeiten von Adamzik (2012) ${ }^{10}$ und Heer $(2010)^{11}$ hingewiesen. Das Interesse fokussiert sich auf den textlinguistischen Status des Schulbuchs (vgl. hierzu exemplarisch Gansel 2015 ${ }^{12}$ ) und die Vernetztheit von Schulbuch

\footnotetext{
5 E. Zawadzka, Polnische Lehrwerke für Deutsch als Fremdsprache-ein geschichtlicher Überblick. In: Studia Niemcoznawcze, Band 29/2005, S. 603-633.

${ }^{6}$ R. Budziak, Deutsch als Fremdsprache in Polen. Sprachlehrbücher aus dem 16. bis 18. Jahrhundert, Harrasowitz Verlag, Wiesbaden 2011.

7 Ein Überblick über diesbezügliche polnischsprachige Forschung ist z. B. in J. Nocoń, Podręcznik szkolny $w$ dyskursie dydaktycznym - tradycja i zmiana, Uniwersytet Opolski, Opole 009 zu finden.

8 Vgl. J. Kiesendahl, C. Ott (Hrsg.), Linguistik und Schulbuchforschung. Gegenstände - Methoden - Perspektiven, V\&R Unipress, Göttingen 2015, S. 9.

9 Ebenda: 11.

${ }^{10}$ K. Adamzik, Kontrastive Textologie am Beispiel des Schulbuchs. In: tekst i dyskurs / text und diskurs 5, 2012, S. 53-91.

${ }^{11}$ N. Heer, Das Schulbuch als textlinguistischer Forschungsgegenstand. In: Foschi, A.M./Hepp, M. u. a. (Hrsg.): Text und Stil im Kulturvergleich, München 2010, S. 471-481.

12 C. Gansel, Zum Textlinguistischen Status des Schulbuchs. In: Kiesendahl, J./Ott, C. (Hrsg.): Linguistik und Schulbuchforschung, V\&R unipress, Göttingen 2015, S. 111-135.
} 
und anderen Elementen innerhalb des Textsortenverbunds sowie von Schulbuch als zentralem Element des Verbunds und anderen Teilen von Textsortennetzen.

Die zweite Perspektive der Untersuchungen zu Schulbuch, ist die, die das Schulbuch als multimodalen Text bzw. einen Bestandteil eines Bildungsmedienverbunds fasst. In dieser Hinsicht ist allerdings ein großes Forschungsdefizit zu verzeichnen, das möglicherweise im Rahmen der Medienlinguistik, ,einer Subdisziplin in statu nascendi“13, zu bewältigen wäre. Es fehlen bislang linguistisch ausgerichtete Untersuchungen zum Schulbuch als multimodalem Medium, auch wenn die Zahl bildlinguistischer Arbeiten in den letzten Jahren kaum überschaubar ist. Im Kontext dieses Beitrags sei exemplarisch auf den Sammelband Das Bild im Schulbuch von Heinze/Matthes (2010) $)^{14}$ hingewiesen.

Drittens soll hier die diskurslinguistische Perspektive genannt werden, in deren Rahmen das Schulbuch als „Widerspiegelung der jeweils geltenden, diskursiv bedingten Wissensordnung“15 und Schulbücher als „Bausteine von Diskursen“"16 verstanden werden. Untersuchungen im Rahmen dieses Forschungsfeldes konzentrieren sich auf sprachlich vermitteltes soziokulturelles Wissen, das mittels diskurslinguistischen Instrumentariums und derlei Analyseverfahren herausgestellt wird. Auch hier ist anzumerken, dass die Diskurslinguistik eine noch junge Disziplin ist und wohl aus diesem Grunde es noch an systematischen diskurslinguistisch orientierten Untersuchungen zu Schulbüchern fehlt. Nichtsdestotrotz sei hier auf einige Einzelstudien hingewiesen, wie z. B. Dreesen $2015^{17}$, Dreesen/Judkowiak $2011^{18}$, Pfalzgraf $2011^{19}$, Ott $2015^{20}$, Höhne $2010^{21}$.

${ }^{13}$ H.-H. Lüger, Entwicklungen der Medienlinguistik. In: Beiträge zur Fremdsprachenvermittlung, nr 58, 2016, S. 60.

${ }^{14}$ C. Heinze, E. Matthes, (Hrsg.): Das Bild im Schulbuch, Verlag Julius Klinkhardt, Bad Heilbrunn 2010.

${ }_{15} \mathrm{Ph}$. Dreesen, Sprache - Wissen - Kontingenz. Die Kontrastive Diskurslinguistik in der Schulbuchforschung am Beispiel deutscher und polnischer Geschichtsbücher. In: Kiesendahl, J./Ott, C. (Hrsg.): Linguistik und Schulbuchforschung, V\&R unipress, Göttingen 2015, S. 57.

${ }^{16}$ C. Ott, Bildungsmedien als Gegenstand linguistischer Forschung. Thesen, Methoden, Perspektiven. In: Kiesendahl, J./Ott, C. (Hrsg.): Linguistik und Schulbuchforschung. Gegenstände - Methoden - Perspektiven, V\&R unipress, Göttingen 2015, S. 34.

${ }_{17} \mathrm{Ph}$. Dreesen, Sprache - Wissen - Kontingenz. Die Kontrastive Diskurslinguistik in der Schulbuchforschung am Beispiel deutscher und polnischer Geschichtsbücher. In: Kiesendahl, J./Ott, C. (Hrsg.): Linguistik und Schulbuchforschung, V\&R unipress, Göttingen 2015, S 53-83.

${ }_{18} \mathrm{Ph}$. Dreesen, J. Judkowiak, Bierni na Wschodzie, ponoszqcy zbiorowa wine $i$ oczywiście obecni w Europie - krytyka niemieckich i polskich podręczników do historii w oparciu o kontrastywna lingwistyke dyskursu. In: tekst $i$ dyskurs / text und diskurs 5, 2012, S. 93-126.

${ }^{19}$ F. Pfalzgraf, Ausländer, Fremde(s) und Minderheiten in deutschen Fibeln 1933-1945. In: Muttersprache 121, Heft 3/2011, S. 161-192.

${ }^{20}$ C. Ott, Bildungsmedien als Gegenstand linguistischer Forschung. Thesen, Methoden, Perspektiven. In: Kiesendahl, J./Ott, C. (Hrsg.): Linguistik und Schulbuchforschung. Gegenstände - Methoden - Perspektiven, V\&R unipress, Göttingen 2015, S. 19-37.

${ }^{21}$ T. Höhne, Die thematische Diskursanalyse - dargestellt am Beispiel von Schulbüchern. In: Keller, R. u. a. (Hrsg.): Handbuch Sozialwissenschaftliche Diskursanalyse, Bd. 2: Forschungspraxis, Wiesbaden 2010, S. 423-453. 
Obwohl die Forschungslage zu Bildungsmedien im Allgemeinen als „dürftig”22 und „pädagogisch dominiert“ ${ }^{“ 23}$ charakterisiert werden kann, ist seit geraumer Zeit ein wachsendes Interesse an linguistisch fundierten Untersuchungen zu Bildungsmedien zu verzeichnen, das sich in zahlreichen Einzelstudien und auch in größeren Projekten, die u. a. von Georg-Eckert-Institut unterstützt werden, niederschlägt. Einen ausführlichen Überblick über die aktuelle Forschungslage gibt der Sammelband von Kiesendahl und Ott (2015) Linguistik und Schulbuchforschung. Gegenstände - Methoden - Perspektiven. Seine Lektüre bestätigt allerdings die drei Defizite der Schulbuchforschung, die Hoppe ${ }^{24}$ folgendermaßen charakterisiert:

- das »theoretische Defizit« (eine allgemein anerkannte Theorie des Schulbuchs fehlt bislang),

- das »empirische Defizit« (bezogen auf den konkreten Einsatz des Schulbuchs im Unterricht)

- das »methodische Defizit« (Verfahren und Instrumente der Analyse).

Mit Ott kann ebenfalls geschlussfolgert werden, dass Bildungsmedienforschung vor allem als Schulbuchforschung betrieben wird, denn das Schulbuch steht im Zentrum der Bildungsmedienforschung ${ }^{25}$.

\section{FREMDSPRACHENLEHRWERK ALS MEDIUM, MULTIMEDIUM UND MASSENMEDIUM}

Jedes moderne Fremdsprachenlehrwerk umfasst verschiedene Arten von Medien: Printmedien, digitale Medien, Audiomedien und audiovisuelle Medien. Es vereint daher verschiedene Zeichensysteme und besteht aus Verbalem (geschriebene und gesprochene Sprache), Visuellem (Bilder: Zeichnungen, Fotos, Diagramme u.a., sowie Typografie), sowie aus Audialem (gesprochene Sprache [Audiotexte], Geräusche, Lieder, d.h. alle Tonaufnahmen, meistens in Form von CD-Roms) und Audiovisuellem (Ton und Bild und Sprache, z. B. Filme als DVD-Roms, auch Nonverbales, Musik). Zwischen den einzelnen Kodes gibt es direkte bzw. indirekte Beziehungen, die sich $u$. a. auch durch direkte sprachliche Verweise im Lehrwerk manifestieren und als intertextuelle Bezüge par excellence aufgefasst werden können. Niemand kann sich heute ein Fremdsprachenlehrwerk ohne Tonaufnahmen oder ohne Bilder vorstellen. Genauso selbstverständlich erscheinen heute gewisse Einteilungen in Kursbuch, Arbeitsbuch, Lehrerhandreichungen etc. In Anlehnung an Lüger ${ }^{26}$

${ }^{22}$ D. Wrobel, A. Müller, A. (Hrsg.): Bildungsmedien für den Deutschunterricht. Vielfalt, Entwicklungen, Herausforderungen, Verlag Julius Klinkhardt, Bad Heilbrunn 2013, S 8.

23 W. Wiater (Hrsg.), Schulbuchforschung in Europa - Bestandsaufnahme und Zukunftsperspektive, Verlag Julius Klinkhardt, Bad Heilbrunn 2003, S. 11.

${ }^{24} \mathrm{Vgl}$. H. Hoppe, Schreiben in Unterrichtswerken. Eine qualitative Studie über die Modellierung der Textsorte Bericht in ausgewählten Unterrichtswerken sowie den Einsatz im Unterricht, Peter Lang, Frankfurt am Main 2011, S. 28.

25 Vgl. C. Ott, Bildungsmedien als Gegenstand linguistischer Forschung. Thesen, Methoden, Perspektiven. In: Kiesendahl, J./Ott, C. (Hrsg.): Linguistik und Schulbuchforschung. GegenständeMethoden - Perspektiven, V\&R unipress, Göttingen 2015, S. 20.

${ }^{26}$ H.-H. Lüger, Entwicklungen der Medienlinguistik. In: Beiträge zur Fremdsprachenvermittlung, Nr. 58, 2016, S. 59-91. 
können Lehrwerke als Medientexte (Wallsten spricht hier von multimodalem Text $^{27}$ ) betrachtet werden. Ihre spezifischen Eigenschaften sind die, die allen Medientexten zukommen: eine hohe semantische Komplexität, d.h. Elemente verschiedener Zeichensysteme (Kodes), die sich gegenseitig beeinflussen, ergänzen, vereindeutigen können. Sie sind ein multikodales (multimodales) Gesamtprodukt. Die Wirkung dieses Gesamtprodukts ergibt sich aus dem Zusammenspiel verbaler, visueller, audialer, audiovisueller Komponenten ${ }^{28}$. Dieses Zusammenspiel aller Kodes ist aber nicht ganz beliebig und seine Wahrnehmung und Verarbeitung ist nicht ganz dem Zufall und dem (Un)Willen der Lernenden überlassen. Die Rollen, Funktionen, d.h. die Relevanz, die den einzelnen Komponenten eines Bildungsmediums zukommt, sind meistens vorgeplant und vorgegeben. Auf manche Komponenten kann unter Umständen verzichtet werden, wenn auch nie ganz, aber im Grunde sind alle in Anspruch zu nehmen, denn nur dann kann sich der didaktische Erfolg einstellen. Daher kann das Fremdsprachenlehrwerk nicht nur als ein Medienverbund, sondern tatsächlich als ein Multimedium - ein Medium, das mehrere miteinander verflochtene Medien einbezieht, deren Inanspruchnahme den Lernerfolg herbeiführt, bezeichnet und verstanden werden. Darin besteht auch seine Spezifik im Unterschied zu anderen Lehrbüchern bzw. Bildungsmedien, die in anderen Fächern als Fremdsprachenunterricht (FU) eingesetzt werden. Da es im FU darum geht, bei den Lernenden die vier Grundfertigkeiten: Sprechen, Hören, Lesen und Schreiben in einer Fremdsprache zu entwickeln und zu fördern, sind alle Modalitäten/Kodes des Multimediums unabdingbar. Ihr Zusammenspiel ist didaktisch durchdacht und oft sind die mithilfe eines Zeichensystems vermittelten Inhalte eine notwendige Voraussetzung für das Erlernen von Inhalten, die mithilfe eines anderen Zeichensystems vermittelt werden (hörendes Verstehen als Voraussetzung für die Lektüre eines Textes oder die Lösung einer schriftlich zu bewältigenden Aufgabe etc.). Das Fremdsprachenlernen geschieht im ständigen Wechseln von Modalitäten.

Für den hier untersuchten Gegenstand gelten auch alle Lesarten der Bezeichnung Medium. Ein Fremdsprachenlehrwerk ist ein Medium im technologischen Sinne ${ }^{29}$. Es ist eine Kommunikationsform, ein Informationsträger und ein „Medium des Kulturtransfers“ ${ }^{\text {“30 }}$. Als Kommunikationsform ist es aber keine ,gespeicherte Einweg-Schriftkommunikation mehr" ${ }^{31}$, sondern ein interaktives Medium für den interaktiven Unterricht.

Die einzelnen Zeichenarten (Modalitäten) können ebenfalls als Medien gedeutet werden (Sprache als Medium, Schrift als Medium, Bild als Medium, Ton als Medium).

${ }^{27}$ B. Wallsten, An der Schnittstelle zwischen Bild und Text. Bildunterschriften in Geschichtslehrbüchern als Untersuchungsgegenstand sprachwissenschaftlicher Schulbuchforschung. In Kiesendahl, J./Ott, C. (Hrsg.): Linguistik und Schulbuchforschung. Gegenstände - Methoden - Perspektiven, V\&R unipress, Göttingen 2015, S. 137.

28 Vgl. H.-H. Lüger, Entwicklungen der Medienlinguistik. In: Beiträge zur Fremdsprachenvermittlung, Nr. 58, 2016, S. 61.

${ }^{29}$ „Medien sind konkrete, materielle Hilfsmittel, mit denen Zeichen verstärkt, hergestellt, gespeichert und/oder übertragen werden können“ (W. Holly, Zur Rolle der Sprache in Medien. In: Muttersprache 107 (1997), S. 69).

${ }^{30}$ Zum Schulbuch als Medium des Kulturtransfers vgl. N. Heer, Das Schulbuch als textlinguistischer Forschungsgegenstand. In: Foschi, A.M./Hepp, M. u. a. (Hrsg.): Text und Stil im Kulturvergleich, München 2010, S. 474.

31 So Holly über das Medium und die Kommunikationsform Buch in W. Holly, Zur Rolle der Sprache in Medien. In: Muttersprache 107 (1997), S. 69. 
Das Schulbuch kann auch als „Massenmedium” angesehen werden: es ist institutionalisiert, richtet sich an disperses Publikum, es ist multimodal, funktional, zwischen dem Autor/Herausgeber und Lesern gibt es eine räumliche Distanz. Das Schulbuch wird auch für Prototyp eines Bildungsmediums gehalten, so $\mathrm{Ott}^{32}$. Die Herausgeberinnen der bereits erwähnten Publikation aus dem Bereich der germanistischen Schulbuchforschung Kiesendahl und Ott (2015) definieren das Schulbuch als Bündelung und Transmitter schulrelevanten Wissens, d. h. auch als Medium (Transmitter) im Verbund (Bündelung): „Das Schulbuch gilt als Bündelung und Transmitter schulrelevanten Wissens für und in den Unterricht und wird in diesem Sinn als Fenster ins Unterrichtsgeschehen und als Wissensstandsanzeige für das im Unterricht Gelehrte und zu Lernende verstanden",33.

\section{ENTWICKLUNG DES MEDIUMS SCHULBUCH ZUM MODERNEN BILDUNGSMEDIUM}

Bevor auf die Ergebnisse der Analyse moderner und älterer Schulbucher eingegangen wird, soll noch kurz darauf hingewiesen werden, dass auf die Form und den Inhalt von Schulbüchern viele Faktoren Einfluss haben (können). Es ist also nicht ohne Bedeutung, wie sich die Rahmenbedingungen des Lehr- und Lernens im hier analysierten Zeitraum, d.h. in den letzten dreißig Jahren, verändert haben.

Die wichtigsten Faktoren, die die Lernumgebung beeinflusst haben, sind nach Dakowska $^{34}$ und Adamzik ${ }^{35}$ gesellschaftliche sowie technologische und bildungspolitische Veränderungen. Hier werden sie kurz aufgelistet:

- Wir sind Informationsgesellschaft bzw. Wissensgesellschaft geworden: Wissen wurde zur Ware. Fremdsprachenkenntnisse gelten heute als selbstverständlich, wobei dem Englischen die Funktion der lingua franca zukommt. Das Fremdsprachenlernen wurde zu einer Massenerscheinung (Egalitarismus). Auf dem Markt trifft die große Nachfrage nach Bildungsmedien auf ein ebenfalls reiches Angebot an Bildungsmedien. Der Konkurrenzdruck unter Herstellern (Verlagen) ist groß.

- Technologische Entwicklung mit Schwerpunkt elektronische Massenmedien machte unseren Planeten zum global village: Wir agieren in einer globalen Kultur, denn durch das Internet überwinden wir Zeit- und Raumgrenzen in der Kommunikation. Interkulturelle Kommunikation wird zur Norm und stellt keinen Einzelfall dar. Die technische und technologische Entwicklung trägt dazu bei, dass „alte“ im Sinne traditionelle, schwarz-weiße Schulbücher in Papierform durch neue Formen ersetzt werden.

- Gesellschaftliche und technologische Veränderungen sowie neue Forschungserkenntnisse bedingen die Entwicklung der Fremdsprachendidaktik: immer wieder

${ }^{32}$ C. Ott, Bildungsmedien als Gegenstand linguistischer Forschung. Thesen, Methoden, Perspektiven. In: Kiesendahl, J./Ott, C. (Hrsg.): Linguistik und Schulbuchforschung. Gegenstände - Methoden - Perspektiven, V\&R unipress, Göttingen 2015, S. 20.

33 J. Kiesendahl, C. Ott, (Hrsg.): Linguistik und Schulbuchforschung. Gegenstände - Methoden Perspektiven, V\&R Unipress, Göttingen 2015, S. 9.

${ }^{34} \mathrm{Mehr}$ dazu vgl. exemplarisch M. Dakowska, O rozwoju dydaktyki języków obcych jako dyscypliny naukowej, Warszawa 2015, S. 47.

35 Vgl. dazu ebenfalls K. Adamzik, Kontrastive Textologie am Beispiel des Schulbuchs. In: tekst i dyskurs / text und diskurs 5, 2012, S. 58 f. 
entstehen neue Lehr- und Lernmethoden bzw. die bereits existierenden werden weiterentwickelt und modernisiert.

- Von den gesellschaftlichen und technologischen Veränderungen waren (und sind) alle Lebensbereiche betroffen, darunter auch das Bildungswesen (u.a. PISA, Bologna-Prozess), in dessen Rahmen die Fremdsprachendidaktik in Europa einem besonderen Internationalisierungsdruck ausgesetzt wird. Auch Verlage agieren global. Hierunter fallen auch Maßnahmen zur Evaluation und Qualitätssicherung im Bildungsbereich: in Bezug auf Fremdsprachendidaktik handelt es sich z. B. um die Einführung von internationalen Standards - dem Europäischem Referenzrahmen, bilinguale Schulen, u.a.

\subsection{Traditionelle und neue Bildungsmedien im Vergleich}

Im Rahmen der Analyse wurde ,das didaktische Massenmedium der 80er Jahre“ Lernt mit uns von J. Honsza und N. Honsza mit dem aktuell in den Mittelschulen in Polen eingesetzten Lehrwerk Infos ${ }^{36}$ von C. Serzysko, B. Sekulski, N. Drabich, T. Gajownik verglichen. Von Interesse waren solche Kriterien wie: Aufbau, graphische Gestaltung, Auswahl von Texten, Kompetenzenaufbau und die „Zusammensetzung“ des Lehrwerks, d. h. die einzelnen Bestandteile des Lehrmittels. In Bezug auf weitere Ausführungen gilt es an dieser Stelle anzumerken, dass es in diesem Beitrag nicht um eine ausführliche Analyse hinsichtlich eines jeden Kriteriums geht, sondern vielmehr darum, den Überblick über die gesamte Entwicklung des untersuchten Gegenstands nicht aus den Augen zu verlieren. Daher werden die einzelnen Kriterien notwendigerweise lediglich zu gewissen Orientierungshilfen, die es erlauben, die erwähnte Entwicklung grob zu skizzieren, ohne auf Einzelheiten einzugehen.

Das Lehrbuch von J. Honsza, N. Honsza Lernt mit uns I, II,III, IV (WSiP 1976) wurde sehr zutreffend von Zawadzka beschrieben: Es ,stützte sich auf die sog. cognitiv code learning theory, d.h. neben der Herausbildung der sprachlichen Gewohnheiten und den zu ihnen führenden Automatisierungsübungen legte man einen großen Wert auf die Bewusstmachung der Probleme. Einzelne methodische Einheiten enthielten: Vorübungen (in denen neue Grammatik und Lexik eingeführt wurde), Gespräche (Dialoge als Beispiele praktischer Verwendung), Lesestücke (eigentliche Texte mit polnischer Übersetzung der Vokabeln), Aussprache, Betonung und Satzintonation (Übungen und graphische Darstellungen), Grammatik (Beispielsätze und tabellarische Darstellungen), Übungen (zur Festigung der Grammatik, vor allem Substitutions-, Transformations- und Integrationsübungen), Kontrollaufgaben und Übungen zur Wiederholung sowie Leseecke (Texte zum selbstständigen Lesen). Das Lehrbuch enthielt viele humorvolle Illustrationen“37.

Bezüglich der graphischen Gestaltung wurde bereits darauf hingewiesen, dass das Lehrbuch Illustrationen und nur wenige Fotos erhielt. Das ganze Buch war eigentlich in Schwarz-Weiß und Grauschattierungen und jeder Band hatte noch eine zusätzliche Farbe, um Wichtigeres hervorzuheben.

Das Lehrbuch folgte dem linearen Aufbauprinzip, eine methodische Einheit bedingte die die Darauffolgende und dies bestimmte auch die Art und Weise der Arbeit am Buch und mit dem Buch. Man hat eine Übung nach der anderen gemacht. Es gab keine zusätzlichen

${ }^{36}$ C. Serzysko, B. Sekulski, N. Drabich, T. Gajownik: Infos 1, 2, 3, 4, Longman - Pearson Education 2011.

${ }^{37}$ E. Zawadzka, Polnische Lehrwerke für Deutsch als Fremdsprache - ein geschichtlicher Überblick. In: Studia Niemcoznawcze, Band 29/2005, S. 623. 
Komponenten zum Buch. Das Buch war nicht in die später und auch heute noch üblichen Teile: Kursbuch, Arbeitsbuch (Übungsbuch) aufgeteilt. Es enthielt keine authentischen Texte, sondern Lesetexte, Dialoge und Übungen wurden von den Autoren für die jeweilige Lernstufe verfasst. Diese Texte enthielten kaum oder nur vereinzelt umgangssprachliche Ausdrücke. Zum Buch gab es keine weiteren Komponenten (Bestandteile) weder in Form von Tonaufnahmen (Schallplatten), noch als Dias oder Filme. Es war ein einfaches schwarz-weiß-graues lineares Medium, in dem Bilder eher als ein gewisser Zusatz, eine Art Auflockerung dienten. Wohl aus technischen Gründen war nur der Umschlag bunt (Kostenfaktor).

Stellvertretend für moderne Bildungsmedien, die im aktuellen Schulbetrieb genutzt werden, wird im Weiteren das Lehrwerk Infos präsentiert. Das Kursbuch und das Arbeitsbuch sind hier in einem Band integriert ${ }^{38}$. Es kommen noch CDRoms mit verschiedenen Aufgaben und Ausspracheschulung hinzu. Sowohl der Kursbuch- als auch der Übungsteil enthalten viele Übungen, die nur mit Hilfe des Internets bewältigt werden können, d.h. interaktiv gestaltet sind.

Die graphische Gestaltung steht hier im Dienste der Übersichtlichkeit. Der Kursbuchteil enthält viele bunte Bilder, hauptsächlich Fotos, aber auch Zeichnungen und graphische Darstellungen grammatischer Phänomene. Die Bilder erfüllen verschiedenartige Funktionen und stellen oft einen Sprechanlass dar. Aus einem modernen Lehrbuch sind Bilder und andere graphische Elemente einfach nicht mehr wegzudenken. Der Kursbuchteil ist bunt, aber übersichtlich. Der Übungsteil ist schwarz-grau-weiß gestaltet. Das Titelblatt eines jeden Kapitels im Übungsteil ist eine schwarz-weiße Kopie des jeweiligen bunten Titelblatts aus dem Kursbuchteil.

Die innere Struktur des Buches folgt der Regel: eine Einheit - ein kommunikatives Ziel - eine Doppelseite. Charakteristisch ist ein modularer Aufbau nach dem Cluster-Prinzip und damit eine Anpassung an die mediale Kommunikation (ähnlich Online-Publikationen mit Hypertextstrukturierung und interaktivem Potenzial). Die einzelnen Aufgaben stehen zur Wahl. Bei Bedarf werden sie um die Aufgaben aus dem Übungsteil ergänzt.

Die Texte basieren auf der Alltagslexik. Es sind authentische Texte.

Jedes Kapitel enthält Aufgaben zur Förderung der sprachlichen Fertigkeiten: Hören, Lesen, Sprechen, Schreiben. Daraus ergibt sich eine enge Verbindung mit anderen Elementen des Gefüges (CDs).

Das Lehrwerk Infos ist auch ein komplettes Angebot für Lehrer. Die Lehrerhandreichungen sind dicker (!) als das eigentliche Lehrbuch. Sie enthalten aber noch 3 CDs und einen Testgenerator sowie viele Hinweise bezüglich der einzelnen Lektionen, ergänzende Materialien (Arbeitsblätter als Kopiervorlagen), Kurztests, Kontrollarbeiten, Prüfungssätze (Abitur) und Lösungsschlüssel für alle Aufgaben, Tests, Kontrollarbeiten und Prüfungssätze.

\footnotetext{
${ }^{38}$ Unlängst waren im Fremdsprachenunterricht typischerweise Lehrwerke im Einsatz, die das Kursbuch und das Arbeitsbuch meistens in Form von 2 getrennten Büchern führten. Auch heute ist dies oft der Fall. Inwieweit die beiden Teile unentbehrlich für den didaktischen Prozess sind, ist eine andere Frage. Wegen hoher Preise von (v. a. ausländischen) Fremdsprachenlehrwerken hat man im didaktischen Alltag oft auf den Einsatz von Arbeitsbüchern verzichtet. Möglicherweise ist dies einer der Gründe, warum heute beide Teile wieder zu einem Band zusammengeführt werden.
} 
Moderne Fremdsprachenlehrwerke sind eigentlich „,komplette Fertigprodukte“ für beide Parteien eines didaktischen Prozesses. Der Lernende wird mit allen Hilfsmitteln ausgestattet, um der Aufgabe gerecht zu werden, die Fertigkeiten: Hören, Lesen, Sprechen und Schreiben in einer fremden Sprache multisensorisch unter Nutzung moderner Technologien zu entwickeln. Der Lehrende bekommt ebenfalls fertige Unterrichtsszenarien, manchmal mit ausformulierten Fragen und Aufgaben für den Unterricht, welche der jew. Lernstufe, Gruppe angepasst sind. So wird in den Lehrerhandreichungen zu Mit links! ${ }^{39}$ die betreffende Seite aus dem Lehrbuch abgebildet und in der Abbildung sind die richtigen Lösungen zu den einzelnen Aufgaben markiert. Unterhalb der Abbildung sind fertige Fragen und Aufgaben angegeben, auf die der Lehrer zum stofflichen Aufwärmen der Gruppe zurückgreifen kann. Sie sind allerdings zweigeteilt in die Formulierungen und Fragen, die für eine fortgeschrittenere Gruppe bestimmt sind und in jene, welche für weniger fortgeschrittene Schüler gedacht sind. Der Lehrende, der z.B. mit Infos oder mit Mit links! oder Meine Welttour ${ }^{40}$ u. a. modernen Lehrwerken arbeitet, bekommt Zugang zu Online-Ressourcen für Lehrende $^{41}$, darunter zu Zusatzmaterialien, Filmen, Glossaren, Whiteboard-Software und Übungsangeboten für IWB, aber auch zu Curricula, Richtlinien für die Benotung und Auswertung schulischer Arbeiten, Unterrichtsentwürfen, Planung von Unterrichtsstunden etc. Somit wird er nicht nur für seine Aktivität als Lehrkraft im Unterricht komplett ausgestattet, sondern es wird an seine Verpflichtungen gegenüber der Schule als Institution gedacht und ihm werden Unterlagen zugänglich gemacht, die es möglich machen, diesen Pflichten nachzugehen. Mit anderen Worten: Er bekommt sowohl didaktische als auch amtliche Instrumente samt Anleitung in die Hand und ... „es kann losgehen“.

Die digitalen Versionen von Komponenten eines Bildungsmediums samt Zusatzangeboten für Lehrkräfte stellen m. E. einen wichtigen Vorteil, oft einen besonderen Mehrwert dar, der bestimmt dazu verhilft, sich für die bestimmte Reihe (den bestimmten Titel) als Leitmedium im schulischen Fremdsprachenunterricht zu entscheiden. Die Vollständigkeit des Angebots für beide Parteien des didaktischen Prozesses ergibt sich wohl aus wirtschaftlichen Gründen der Konkurrenz unter den Verlagen. Die monopolistische Stellung von Lernt mit uns ist heute kaum vorstellbar. So wie das heutige Angebot an Bildungsmedien kaum überschaubar ist. Dank technologischer Entwicklung umfasst die Bezeichnung Bildungsmedium verschiedene Formen, die sich unmöglich vollständig auflisten lassen: ,physische und digitale Bücher (Schulbücher, Begleitbände, eigenständige Publikationen für Lehrende, akademische Einführungswerke, Quellensammlungen), Hefte (Arbeitshefte, Lösungshefte, aber auch Schulhefte), lose Materialiensammlungen (z.B. von Lehrkräften zusammengetragene Unterrichtsmaterialien inklusive Tafelbilder und Overheadfolien; Online-Materialsammlungen), Materialien, die im Kern der Veranschaulichung des Lernstoffs dienen (Wandbilder, Karten, Artefakte, Modelle, Präparate, weitere Lehr-Bildmedien (Lehrfilme, Lehrdiareihen und andere, CD-ROMs und DVDs, die über die reine Visualisierung hinaus oft zur spielerischen Interaktion anleiten), Lernspiele, Apps (z.B. mobile

${ }^{39}$ Mit links! Książka nauczyciela von E. Kręciejewska und D. Lisowska (Pearson 2012).

${ }^{40}$ Meine Welttour 1, 2, 3 von Sylwia Mróz-Dwornikowska (Nowa Era 2015).

$41 \mathrm{Vgl}$. Infos: https://www.pearson.pl/niemiecki/epanel-panel-nauczyciela-pearson.html; Meine Welttour: http://www.dlanauczyciela.pl/zasoby/szkoly-ponadgimnazjalne/jezyk-niemiecki/meinewelttour (10.09.2017). 
Sprachtrainer) und weitere virtuelle Lernumgebung (z.B. Online-Tests, Lernportale, interaktive 3D-Animationen für digitale Tafelsysteme"42.

Der inhaltliche Vergleich von Lernt mit uns und Infos bestätigt, dass es inzwischen in Polen zu einer Entideologisierung der Schulbuchinhalte kam. Beiden Medien liegen ganz andere didaktische Konzepte zugrunde. In der Zeit dazwischen kam es im Zuge der audiovisuellen Methode zum Ausbau des Visuellen und Auditiven, gemäß dem Prinzip der Visualisierung und dem kommunikativ-pragmatischen Ansatz. Die visuellen Elemente erfüllen verschiedene kommunikative, typographische und ästhetische Funktionen. Alle stehen grundsätzlich im Dienste der Anschaulichkeit, aber sie können einer besseren Übersichtlichkeit dienen, sowie Aufmerksamkeit und Interesse wecken, d.h. motivieren. Sie stiften Identität und vermitteln Informationen, stimmen die Lerner ein, dienen als Sprechanlass und Identifizierungselement. All das bedeutet, ihnen kommen wichtige psychologische, didaktische und kommunikative Funktionen zu.

Der Einzug des Visuellen und Audiovisuellen sowie Audialen in die Lehrwerke, d.h. die Kopräsenz von Sprache, Bild und Ton, außer dass all die Elemente bestimmte didaktische Zielsetzung verfolgen, leistet ebenfalls einen bestimmten Beitrag zur besseren Anschaulichkeit, Attraktivitätssteigerung, Aufmerksamkeitssteigerung, der Vermittlung von Authentizität usw. Die erwähnte Kopräsenz verschiedener Modalitäten beansprucht verschiedene Lernsinne, erhöht die Behaltensleistung, das Verarbeiten und Speichern von Informationen. Sie ist aber der Grund dafür, dass für ein modernes Lehrwerk ein modularer Aufbau nach dem Cluster-Prinzip typisch ist, was wiederum ein System von Verweisen als Navigationshilfen notwendig macht.

Als Multimedium stellt das Fremdsprachenlehrwerk eine zielgerichtete Verbindung verschiedener Medien zu einer Einheit [Medienverbund] dar, mit sehr starken syntagmatischen Relationen innerhalb des Medienverbunds, d.h. zwischen dem Kursbuch, dem Arbeitsbuch, CDs, DVDs, IWB etc. Im analysierten Zeitraum wurden diese Relationen immer dichter. Gleichzeitig werden andere syntagmatische Relationen zu Paralleltexten: Glossaren, Wörterbüchern, Grammatiken etc. nach wie vor aufrechterhalten und ausgebaut. Als Beweise dieser starken Relationen innerhalb des Medienverbunds können direkte Verweise auf Texte/Aufgaben/Inhalte in anderen Elementen des Verbunds gelten. So wird zum Beispiel der Lerner aufgefordert, um eine bestimmte Aufgabe im Übungsbuch zu lösen, auf Inhalte im Kursbuch oder auf einer CD zurückzugreifen. Dies kann zu Beginn gewisse Orientierungsschwierigkeiten mit sich bringen. Die Einarbeitung kann aufwendig sein. Für den Umgang mit modernen Lehrwerken braucht man als Lerner und Lehrer gewisse Medienkompetenz. Aber die Autoren und Herausgeber sind sich dessen bewusst und messen der Lehr- und Lernerfreundlichkeit viel Bedeutung bei, sodass in vielen modernen Lehrwerken die Navigation komplett vorgegeben ist.

Und last but not least: Um dem Prinzip der Lernerautonomie gerecht zu werden, gibt es in Infos (und in den meisten modernen Lehrwerken) eine Beschreibung von Lernstrategien und Instrumente zur Selbsteinschätzung.

${ }^{42}$ C. Ott, Bildungsmedien als Gegenstand linguistischer Forschung. Thesen, Methoden, Perspektiven. In: Kiesendahl, J./Ott, C. (Hrsg.): Linguistik und Schulbuchforschung. Gegenstände-Methoden-Perspektiven, V\&R unipress, Göttingen 2015, S. 20. 


\subsection{Zusammenschau der Ergebnisse. Abschließende Bemerkungen zum Status des Mediums Lehrwerk heute}

Die nachstehende Tabelle zeigt die Befunde der Analyse in komprimierter Form. Die meisten Ergebnisse wurden in den bisherigen Ausführungen bereits kurz besprochen. Auf einige, die im Kontext des vorliegenden Beitrags wichtig sind, wird noch im Weiteren eingegangen.

Tab. 1. Die wichtigsten Unterschiede zwischen den analysierten Medien (Lehrbuch aus den 80er Jahren und ein modernes Fremdsprachenlehrwerk)

\begin{tabular}{|l|l|}
\hline \multicolumn{1}{|c|}{$\begin{array}{c}\text { Das Lehrbuch für Deutsch als } \\
\text { Fremdsprache in den 80er Jahren }\end{array}$} & \multicolumn{1}{c|}{$\begin{array}{c}\text { Das moderne Lehrwerk für Deutsch } \\
\text { als Fremdsprache }\end{array}$} \\
\hline geringes Angebot (ein Lehrbuch) & eine Vielzahl von Titeln \\
\hline Einzelmedium (Buch) & $\begin{array}{l}\text { Multimedium (Serien/Reihen auf der Basis eines } \\
\text { einheitlichen Konzepts) bestehend meistens aus } \\
\text { einigen medialen Komponenten: Büchern } \\
\text { CDs + DVDs + Online-Angebot + IWB }\end{array}$ \\
\hline $\begin{array}{l}\text { Linearität (Einfachheit der Gestaltung) } \\
\text { unkomplizierter Gebrauch }\end{array}$ & $\begin{array}{l}\text { Modularität / Hypertextstrukturierung / Cluster- } \\
\text { prinzip / Plakativität / visuelle Inszenierung - } \\
\text { Navigationshilfen notwendig } \rightarrow \text { „neue” Medi- } \\
\text { enkompetenz }\end{array}$ \\
\hline Bikodalität (Sprache + Bild) & $\begin{array}{l}\text { Multikodalität (Sprache + Bild + Ton) - neue in- } \\
\text { termediale Bezüge (Relationen) }\end{array}$ \\
\hline schwarz-weiss-grau & Einsatz von Farben (bunte Aufmachung) \\
\hline u. U. Einwegkommunikation & $\begin{array}{l}\text { Interaktives Medium (Mehrfachadressierung) } \\
\text { Handlungsanleitung (Lernangebot - punktuelle } \\
\text { Vutzung) }\end{array}$ \\
\hline Autorentexte (artifiziell) & $\begin{array}{l}\text { Authentische Texte (meistens didaktisiert oder } \\
\text { gekürzt) }\end{array}$ \\
\hline keine / wenige Zusatzmaterialien & $\begin{array}{l}\text { ein komplettes Angebot (auch im kommerziel- } \\
\text { len Sinne) für Lehrkraft und Lernende }\end{array}$ \\
\hline $\begin{array}{l}\text { evtl. Medien (Schallplatten, Kassetten) nur für } \\
\text { Lehrer / separat zu kaufen }\end{array}$ & $\begin{array}{l}\text { CDs, DVDs als integrale Bestandteile des Pro- } \\
\text { dukts „Fremdsprachenlehrwerk“ }\end{array}$ \\
\hline Leitendes Medium & $\begin{array}{l}\text { Immer noch wichtig, aber subsidiär zum Unter- } \\
\text { richt (Lerner- und Lehrerautonomie). Keine zu- } \\
\text { verlässigen Angaben über die tatsächliche Nut- } \\
\text { zung. Das Buch ist ein Element von vielen. }\end{array}$ \\
\hline
\end{tabular}

Die Entwicklung des (relativ) einfachen linearen bikodalen (Sprache + Bild in Form von Zeichnungen enthaltenden) schwarz-weißen Mediums Buch der 80er Jahre zu einem modular aufgebauten multikodalen (Sprache + Bild + Ton) Multimedium bunter Aufmachung, das aus einigen Komponenten (Medien) besteht und kein Einwegkommunikation-Medium darstellt, sondern als interaktives Medium konzipiert ist und als Lehrwerk bezeichnet wird, ist zweifelsohne anerkennenswert. Diese Entwicklung regt aber dazu an, nach dem aktuellen Status des Lehrwerkes und insbesondere des Lehrbuches innerhalb des Multimediums zu fragen. 
Früher galt ein Lehrbuch als Medium zur Vermittlung von Wissen. Es handelte sich v. a. um deklaratives Wissen zur rezeptiven Aneignung. Heute (und dies betrifft insbesondere die Sprachlehrbücher, aber nicht nur sie) ist das Lehrbuch eine Handlungsanleitung (es vermittelt deklaratives, aber auch prozedurales Wissen), um kommunikativ in der Interaktion mit anderen Schülern, Lehrern, Kommunikationspartnern erfolgreich zu sein. Deswegen basieren moderne Fremdsprachenlehrwerke auf der Alltagssprache mit umgangssprachlichen Elementen und auf authentischen Texten.

Was kann also heute angesichts der beschriebenen Entwicklung zum Status des Lehrbuchs gesagt werden? „Schulbücher gelten nach wie vor als das wichtigste Bildungsmedium im schulischen Kontext. [...] Gleichzeitig zeigt die Unterrichtserfahrung, dass oftmals auch Kopiervorlagen und Arbeitsblätter zur Vermittlung schulischer Inhalte herangezogen werden.”, so Reuter ${ }^{43}$. Viele Forscher vertreten die Auffassung, dass das Schulbuch (zumindest) -,,eine konstante Größe" ${ }^{44}$, „,eine feste Struktur im Unterrichtsbetrieb”45 oder ein „Leitmedium des Unterrichts" 46 ist.

Eine mögliche Antwort auf die oben gestellte Frage kann in Bezug auf das Fremdsprachenlehrwerk lauten: nach wie vor ist es ein Leitmedium, aber es steht nicht mehr für ein separates Lehrbuch, sondern für ein Multimedium, dessen integraler Bestandteil das Lehrbuch ist. Auf der anderen Seite ist die Vorstellung eines Fremdsprachenkurses ohne Buch irgendwie immer noch ungewöhnlich.

Meines Erachtens hat Peyer Recht, indem sie betont, dass heutzutage der Unterricht als kommunikativer Prozess leitend und das Buch subsidiär zum Unterricht ist ${ }^{47}$. Sie plädiert auch dafür, dass man ein sprachliches Signal setzen könnte, das der beschriebenen Entwicklung Rechnung tragen würde: man solle nicht mehr vom Schulbuch, ,,sondern vom Lehrmittel [sprechen], um bewusst zu machen, dass es nicht um das eine Buch geht, über das sich Lernende den Kopf zerbrechen, sondern um ein Angebot von Texten aller Art, die ihre Funktion in Lernprozessen immer neu entfalten“48. Dies ist noch um den Hinweis zu ergänzen, dass diese Texte dermaßen komplex (im Sinne multikodal) sind und mithilfe verschiedener Medien (im technologischen Sinne) zugänglich gemacht und genutzt werden, dass man tatsächlich von einem Medienverbund bzw. einem Multimedium oder Bildungsme-

${ }^{43}$ C. Reuter, Vernetzt? Schulheft und Schulbuch im Vergleich. In: Kiesendahl, J./Ott, C. (Hrsg.): Linguistik und Schulbuchforschung. Gegenstände - Methoden - Perspektiven, V\&R unipress, Göttingen 2015, S. 177.

${ }^{44}$ C. Gansel, Zum Textlinguistischen Status des Schulbuchs. In: Kiesendahl, J./Ott, C. (Hrsg.): Linguistik und Schulbuchforschung, V\&R unipress, Göttingen 2015, S. 125.

45 Ebenda.

46 B. Wallsten, An der Schnittstelle zwischen Bild und Text. Bildunterschriften in Geschichtslehrbüchern als Untersuchungsgegenstand sprachwissenschaftlicher Schulbuchforschung. In Kiesendahl, J./Ott, C. (Hrsg.): Linguistik und Schulbuchforschung. Gegenstände - Methoden - Perspektiven, V\&R unipress, Göttingen 2015, S. 139.

${ }^{47}$ Vgl. A. Peyer, Wünsche der Sprachdidaktik an die Schulbuchforschung. In: Kiesendahl, J./Ott, C. (Hrsg.): Linguistik und Schulbuchforschung. Gegenstände-Methoden - Perspektiven, V\&R unipress, Göttingen 2015, S. 319.

${ }^{48}$ Ebenda: 335. 
dium sprechen sollte. Die Bezeichnung Buch bzw. Schulbuch ist in Bezug auf das Fremdsprachenlehrwerk sehr ungenau ${ }^{49}$.

\section{SCHLUSSBEMERKUNGEN}

Die beschriebenen Entwicklungen zeugen von

- einem Anstieg der Multimedialität

Gemeint ist die Tendenz zur Verdichtung syntagmatischer Relationen innerhalb des Medienverbunds. Alle Bestandteile des Multimediums sind unersetzlich.

- einem Anstieg der Multikodalität Tendenz zur Komplexität: Kein Fremdsprachenlehrwerk ohne Ton, Bild, Text.

- $\quad$ einem Anstieg der Digitalisierung Tendenz zur weiteren Technisierung des Unterrichts: der Zugang zum Internet wird unentbehrlich.

Im Kontext der obigen Ausführungen tauchen auch bestimmte Forschungslücken auf: Bereits 2010 bemerkte Heer: „Schulbücher sind Musterbeispiele für Intertextualität, die auf mehreren Ebenen auftreten kann“50.

Analog dazu kann geschlussfolgert werden:

- Schulbücher (eigentlich moderne Lehrwerke, d.h. Bildungsmedien) sind Musterbeispiele für Multimodalität, die zwischen verschiedenen Zeichensystemen auftreten kann.

- Schulbücher (eigentlich Lehrwerke / Bildungsmedien) sind Musterbeispiele für Multimedialität und intermediale Bezüge, die zur Entstehung eines Multimediums führen.

Diese Relationen herauszustellen und zu beschreiben wäre eine Aufgabe der Medienlinguistik.

Wenn man sich die Entwicklung des Mediums Schulbuch/Fremdsprachenlehrwerk in den letzten dreißig, vierzig Jahren vor Augen führt, kommt man nicht um die Frage umhin: Wie wird das Fremdsprachenlernen und -lehren in der Zukunft aussehen? Wird man noch aus Büchern in Papierform lernen? Vielleicht werden digitale Bücher Übergewicht haben? Oder aber werden Schulbücher marginalisiert und durch eine erweiterte bzw. virtuelle Wirklichkeit weggedrängt? Im Moment lassen sich diese Fragen nicht eindeutig beantworten. Einer Sache können wir aber sicher sein: Es wird Neues kommen, denn nichts ist so beständig, wie der Wandel.

49 Auch K. Adamzik schreibt: „Neue Lehrmaterialien sind in so starkem Ausmaß von diesen Entwicklungen [Multimedialität und De-Linearisierung - M. S.] betroffen, dass es kaum noch gerechtfertigt erscheint, den Ausdruck Schul-Buch überhaupt als Kennmarke zu verwenden." (K. Adamzik, Kontrastive Textologie am Beispiel des Schulbuchs. In: tekst i dyskurs / text und diskurs 5, 2012, S. 55).

${ }^{50}$ N. Heer fasst Schulbuch als ein Teil eines komplexen Systems der Unterrichtsmittel auf (Vgl. N. Heer, Das Schulbuch als textlinguistischer Forschungsgegenstand. In: Foschi, A.M./Hepp, M. u. a. (Hrsg.): Text und Stil im Kulturvergleich, München 2010, S. 474). 


\section{LITERATUR}

[1] Adamzik K., Kontrastive Textologie am Beispiel des Schulbuchs. In: Tekst i dyskurs / Text und diskurs 5, 2012.

[2] Budziak R., Deutsch als Fremdsprache in Polen. Sprachlehrbücher aus dem 16. bis 18. Jahrhundert, Harrasowitz Verlag, Wiesbaden 2011.

[3] Dakowska M., O rozwoju dydaktyki języków obcych jako dyscypliny naukowej, Wydawnictwo Uniwersytetu Warszawskiego, Warszawa 2015.

[4] Dreesen Ph., Sprache - Wissen - Kontingenz. Die Kontrastive Diskurslinguistik in der Schulbuchforschung am Beispiel deutscher und polnischer Geschichtsbücher. In: Kiesendahl J./Ott C. (Hrsg.), Linguistik und Schulbuchforschung, V\&R unipress, Göttingen 2015.

[5] Dreesen Ph./Judkowiak, J., Bierni na Wschodzie, ponoszacy zbiorowa winę i oczywiście obecni w Europie - krytyka niemieckich i polskich podręczników do historii $w$ oparciu o kontrastywną lingwistykę dyskursu. In: Tekst i dyskurs / Text und diskurs 5, 2012.

[6] Gansel C., Zum Textlinguistischen Status des Schulbuchs. In: Kiesendahl J./Ott C. (Hrsg.), Linguistik und Schulbuchforschung, V\&R unipress, Göttingen 2015.

[7] Heer N., Das Schulbuch als textlinguistischer Forschungsgegenstand. In: Foschi A.M./Hepp M. u. a. (Hrsg.), Text und Stil im Kulturvergleich, München 2010.

[8] Heinze C./Matthes E. (Hrsg.), Das Bild im Schulbuch, Verlag Julius Klinkhardt, Bad Heilbrunn 2010.

[9] Holly W., Zur Rolle der Sprache in Medien. In: Muttersprache 107, 1997.

[10] Höhne T., Die thematische Diskursanalyse - dargestellt am Beispiel von Schulbüchern. In: Keller R. u. a. (Hrsg.), Handbuch Sozialwissenschaftliche Diskursanalyse, Bd. 2: Forschungspraxis, Wiesbaden 2010.

[11] Hoppe H., Schreiben in Unterrichtswerken. Eine qualitative Studie über die Modellierung der Textsorte Bericht in ausgewählten Unterrichtswerken sowie den Einsatz im Unterricht, Peter Lang, Frankfurt am Main 2011.

[12] Kiesendahl J./Ott C. (Hrsg.), Linguistik und Schulbuchforschung. Gegenstände-Methoden - Perspektiven, V\&R Unipress, Göttingen 2015.

[13] Lüger H.-H., Entwicklungen der Medienlinguistik. In: Beiträge zur Fremdsprachenvermittlung, nr 58, 2016.

[14] Nocoń J., Podręcznik szkolny w dyskursie dydaktycznym - tradycja i zmiana, Uniwersytet Opolski, Opole 2009.

[15] Ott C., Bildungsmedien als Gegenstand linguistischer Forschung. Thesen, Methoden, Perspektiven. In: Kiesendahl J./Ott C. (Hrsg.), Linguistik und Schulbuchforschung. Gegenstände - Methoden - Perspektiven, V\&R unipress, Göttingen 2015.

[16] Pfalzgraf F., Ausländer, Fremde(s) und Minderheiten in deutschen Fibeln 1933-1945. In: Muttersprache 121, Heft 3/2011.

[17] Reuter C., Vernetzt? Schulheft und Schulbuch im Vergleich. In: Kiesendahl J./Ott, C. (Hrsg.), Linguistik und Schulbuchforschung. Gegenstände - Methoden - Perspektiven, V\&R unipress, Göttingen 2015.

[18] Wallsten B., An der Schnittstelle zwischen Bild und Text. Bildunterschriften in Geschichtslehrbüchern als Untersuchungsgegenstand sprachwissenschaftlicher Schulbuchforschung. In Kiesendahl J./Ott, C. (Hrsg.), Linguistik und Schulbuchforschung. Gegenstände - Methoden - Perspektiven, V\&R unipress, Göttingen 2015. 
[19] Wiater W. (Hrsg.), Schulbuchforschung in Europa - Bestandsaufnahme und Zukunftsperspektive, Verlag Julius Klinkhardt, Bad Heilbrunn 2003.

[20] Wrobel D./Müller A. (Hrsg.), Bildungsmedien für den Deutschunterricht. Vielfalt, Entwicklungen, Herausforderungen, Verlag Julius Klinkhardt, Bad Heilbrunn 2013.

[21] Zawadzka E., Polnische Lehrwerke für Deutsch als Fremdsprache - ein geschichtlicher Überblick. In: Studia Niemcoznawcze, Band 29/2005.

\section{O NAJNOWSZYCH TENDENCJACH ROZWOJOWYCH MEDIÓW EDUKACYJNYCH. NOWOCZESNE I TRADYCYJNE PODRECZNIKI SZKOLNE - NIEDEUGA DROGA OD KSIĄŻKI DO MEDIUM EDUKACYJNEGO}

Artykuł stanowi próbę naszkicowania historii medium edukacyjnego - podręcznika szkolnego w ostatnich trzydziestu - czterdziestu latach na podstawie rozwoju podręczników do nauki języka niemieckiego jako obcego. W większości przypadków podręcznik do nauki języka obcego stanowi dziś kompleks multimodalny. Można uznać go również za zjawisko intermedialne. Za punkt wyjścia w niniejszych rozważaniach służy analiza podręcznika Lernt mit uns, który był powszechnie używany w polskich szkołach średnich do nauki języka niemieckiego w latach osiemdziesiątych ubiegłego stulecia i stanowił swoiste 'dydaktyczne mass medium'. Podręcznik ten porównany zostanie z nowoczesnym medium - podręcznikiem Infos, obecnie popularnym w szkołach średnich. Największą zauważalną i najbardziej znaczącą różnicę stanowi przekształcenie się dwukodowego medium książka w wieloczęściową multikodową konstrukcję. Chodzi przy tym nie tylko o zmiany w wyglądzie, treści i strukturze podręczników i materiałów do nauki języków obcych w ciągu ostatnich trzydziestu lat. W artykule porusza się również kwestię aktualnego statusu i specyfiki współczesnych mediów edukacyjnych, przy czym główna uwaga skupia się na multimodalności, 'mediowości' i relacjach intermedialnych. W rozważaniach zaprezentowano również obecny stan badań lingwistycznych (głównie germanistycznych) nad podręcznikami szkolnymi i sformułowano dezyderaty badawcze.

Słowa kluczowe: podręcznik szkolny, medium edukacyjne, multimedium, multimodalność, relacje intermedialne, nauka języków obcych, język niemiecki jako obcy, nauka języka niemieckiego jako obcego.

\section{MODERN AND TRADITIONAL COURSE BOOKS - A SHORT WAY FROM THE SCHOOL BOOK TO THE EDUCATIONAL MEDIUM. ABOUT THE MOST RECENT DEVELOPMENTAL TENDENCIES OF EDUCATIONAL MEDIA}

The aim of this article is to attempt to outline the history of educational medium - the course book within the last thirty-forty years on the basis of the development of course books for teaching German as a foreign language. In most cases, the textbook to teach a foreign language is now the multimodal complex. It can be also treated as the intermedial phenomenon. As the starting point in the dissertation below, the analysis of the course book Lernt mit uns serves. This course book was commonly used in Polish secondary schools to teach German in the eighties of the last century and it was the peculiar 'didactic mass medium'. This textbook will be compared with the modern medium-Infos course book, which has recently been very popular in secondary schools. The most noticeable and the most significant difference is the transformation of two-code media book in the multi-partile, multi-code structure. It's not only a matter of some changes in the look, the context, the structure of the course book and the materials to teach a foreign language within the last 30 years. In this article the issue of current 
status and specificity of modern educational medium is also mentioned, at the same time, the main attention is paid to multimodality, 'mediability 'and intermedia relations. In the dissertation, the present state of the linguistic research (mainly research in German studies) on school books was also presented and the research desiderata were formulated.

Keywords: course book, textbook, school book, educational medium, multimedium, multimodality, intermedia relations, teaching foreign languages, GFL, teaching GFL.

DOI: $10.7862 /$ rz.2017.hss.74

Przestano do redakcji: październik 2017 r.

Przyjęto do druku: grudzień 2017 r. 
